\title{
Impacto do isolamento social na sociedade: Recomendações em tempos de COVID-19
}

Impact of social isolation on society: Recommendations in times of COVID-19

Impacto del aislamiento social en la sociedad: recomendaciones en tiempos de COVID-19

Rayssa Stéfani Sousa Alves

ORCID: https://orcid.org/0000-0002-9666-675X Pontifícia Universidade Católica de Goiás, Brasil E-mail: rayssastefani02@gmail.com

Mariana Pereira Barbosa Silva ORCID: https://orcid.org/0000-0003-0852-8099 Universidade Estadual do Piauí, Brasil E-mail: marianapbsilvaa@gmail.com

Airton César Leite

ORCID: https://orcid.org/0000-0001-7184-8488

Centro Universitário Santo Agostinho, Brasil E-mail: ainton.cesar2014@gmail.com João Kelson Araújo da Silva

ORCID: https://orcid.org/0000-0002-1173-1752 Universidade Federal do Piauí, Brasil

E-mail: joaokelaonjk@gmail.com

Filipe Eugênio Rodrigues Silvestre ORCID: https://orcid.org/0000-0002-3002-1051

Universidade Estadual do Ceará, Brasil E-mail: filipe-eugenio@hotmail.com

Lucília da Costa Silva

ORCID: https://orcid.org/0000-0001-9386-5684

Centro Universitário Santo Agostinho, Brasil E-mail: luciliafiso@outlook.com

Guilia Rivele Souza Fagundes ORCID: https://orcid.org/0000-0003-1834-8278

Universidade do Estado da Bahia, Brasil E-mail: guilia_matina@hotmail.com

Ana Graziela Soares Rêgo Lobão ORCID: https://orcid.org/0000-0003-3003-954X

Centro Universitário Uninovafapi, Brasil

E-mail: grazielarego@hotmail.com

Yago Rodrigues Dalben

ORCID: https://orcid.org/0000-0002-3029-6442

Universidade Federal do Espírito Santo, Brasil

E-mail: yago.rd@hotmail.com

Tamara Saraiva de Assis

ORCID: https://orcid.org/0000-0002-1624-2755 Universidade Federal do Vale do São Francisco, Brasil E-mail: tamsaraiva2@gmail.com

Diego Felipe Borges Aragão

ORCID: https://orcid.org/0000-0002-3626-7087 Universidade Estadual do Piaú, Brasil

E-mail: diego_contemporanea@hotmail.com

Matheus da Silva Paulo

ORCID: https://orcid.org/0000-0002-0021-8240

Universidade Potiguar, Brasil

E-mail: matheus.paulo999@gmail.com

Marilene de Sousa Oliveira

ORCID: https://orcid.org/0000-0002-3204-0686 Universidade Federal do Piauí, Brasil

E-mail: marilene9oliveira@gmail.com

Marciele de Lima Silva

ORCID: https://orcid.org/0000-0003-2827-5316 Instituto de Educação Superior da Paraíba, Brasil

E-mail: marcieledelsilva@gmail.com

Fabiana Neves Lima

ORCID: https://orcid.org/0000-0002-1894-6529 Universidade Estadual do Piauí, Brasil

E-mail: fabiananeves@ifpi.edu.br 


\author{
Bruna Kely Oliveira Santos \\ ORCID: https://orcid.org/0000-0002-6938-5055 \\ Universidade da Integração Internacional da Lusofonia Afro-Brasileira, Brasil \\ E-mail: brunakely40@gmail.com \\ Maria Gabriela Tirelli de Freitas \\ ORCID: https://orcid.org/0000-0002-8103-7280 \\ Universidade Federal de Lavras, Brasil \\ E-mail: Maria.freitas1@estudante.Ufla.br
}

\begin{abstract}
Resumo
Este estudo teve como objetivo identificar o impacto do isolamento social em tempos de COVID-19, e relatar as principais estratégias de combate à doença. Trata-se, de um estudo de revisão integrativa de literatura, com abordagem qualitativa, realizado por meio da busca de artigos indexados nas seguintes bases de dados: Scientific Electronic Library Online (SCIELO), National Center for Biotechnology Information (PUBMED), Literatura Latino-Americana e do Caribe em Ciências da Saúde (LILACS). Os critérios de inclusão compreendem pesquisas de revisão da literatura compreendendo o ano de 2020. Os critérios de exclusão, foram artigos duplicados, incompletos, resumos, resenhas, debates, e relato de experiência. Foram analisados 05 artigos e 04 estudos referentes à entidades de saúde. Os resultados do estudo constataram que, são várias as estratégias e recomendações que visam combater e prevenir a disseminação da COVID-19. Portanto, até o momento, não existem medicamentos ou outras medidas terapêuticas para atuar na erradicação da doença. Várias pesquisas têm sido desenvolvidas nesse sentido, assim como a avaliação e investigação da eficácia de medicamentos já existentes, para atuação no combate ao novo coronavírus. Concluímos que, as medidas preventivas à COVID - 19, ainda não são suficientes para o combate da pandemia. Portanto algumas estratégias preventivas apresentam efetividade na redução do número de casos suspeitos e confirmados da doença, devido o limitado contato com a sociedade, resultando na baixa capacidade de transmissão do vírus.
\end{abstract}

Palavras-chave: Infecções por coronavirus; Prevenção de doenças; Isolamento social.

\begin{abstract}
This study aimed to identify the impact of social isolation in times of COVID-19, and to report the main strategies to combat the disease. This is an integrative literature review study, with a qualitative approach, carried out by searching for articles indexed in the following databases: Scientific Electronic Library Online (SCIELO), National Center for Biotechnology Information (PUBMED), Latin Literature -American and Caribbean Health Sciences (LILACS). The inclusion criteria include literature review surveys covering the year 2020. The exclusion criteria were duplicate, incomplete articles, abstracts, reviews, debates, and experience reports. 05 articles and 04 studies referring to health entities were analyzed. The results of the study found that there are several strategies and recommendations that aim to combat and prevent the spread of COVID-19. So far, there are no drugs or other therapeutic measures to eradicate the disease. Several researches have been developed in this sense, as well as the evaluation and investigation of the effectiveness of existing drugs, to act in the fight against the new coronavirus. We conclude that the preventive measures against COVID - 19 are still not enough to fight the pandemic. Therefore, some preventive strategies are effective in reducing the number of suspected and confirmed cases of the disease, due to limited contact with society, resulting in the low transmission capacity of the virus.
\end{abstract}

Keywords: Coronavirus infections; Disease prevention; Social isolation.

\title{
Resumen
}

Este estudio tuvo como objetivo identificar el impacto del aislamiento social en tiempos del COVID-19 y reportar las principales estrategias para combatir la enfermedad. Se trata de un estudio de revisión bibliográfica integradora, con enfoque cualitativo, realizado mediante la búsqueda de artículos indexados en las siguientes bases de datos: Scientific Electronic Library Online (SCIELO), National Center for Biotechnology Information (PUBMED), Latin Literature Ciencias de la Salud de América y el Caribe (LILACS). Los criterios de inclusión incluyen encuestas de revisión de literatura que cubren el año 2020. Los criterios de exclusión fueron artículos duplicados, incompletos, resúmenes, revisiones, debates e informes de experiencias. Se analizaron 05 artículos y 04 estudios referidos a entidades de salud. Los resultados del estudio encontraron que existen varias estrategias y recomendaciones que tienen como objetivo combatir y prevenir la propagación del COVID-19. Hasta el momento, no existen medicamentos ni otras medidas terapéuticas para erradicar la enfermedad. Se han desarrollado varias investigaciones en este sentido, así como la evaluación e investigación de la efectividad de los fármacos existentes, para actuar en la lucha contra el nuevo coronavirus. Concluimos que las medidas preventivas contra COVID - 19 aún no son suficientes para combatir la pandemia. Por lo tanto, algunas estrategias preventivas son efectivas para reducir el número de casos sospechosos y confirmados de la enfermedad, debido al contacto limitado con la sociedad, lo que resulta en la baja capacidad de transmisión de virus.

Palabras clave: Infecciones por coronavírus; Prevención de enfermedades; Aislamiento social. 


\section{Introdução}

A COVID - 19 (2019-nCov) é um vírus identificado como a causa de um surto de doença respiratória pela primeira vez em Wuhan, China, e teve sua irradiação universal resultando uma pandemia. Os primeiros casos de coronavírus em humanos foram isolados pela primeira vez no século XX, no ano de 1937 (Brasil, 2020).

Contudo, foi em 1965 que o vírus foi nomeado como coronavírus, em sucessão do perfil na microscopia, similar a uma coroa. Os sintomas da COVID-19 é capaz de diversificar de um simples resfriado até uma pneumonia severa. Os sintomas mais comuns são: Tosse, febre, coriza, dor de garganta e dificuldade para respirar (Brasil, 2020).

Segundo a Organização Mundial de Saúde (2020), em dezembro de 2019, um conjunto de casos de pneumonia de etiologia não conhecida, foram notificadas na cidade de Wuhan, província de Hubei (China). Cinco meses após o surgimento dos primeiros casos da doença, a Universidade Johns Hopkins identificou que a COVID-19 já estava instalada em 188 países/regiões, sendo responsáveis por centenas de milhares mortes (Jhu, 2020).

Gorbalenya et al. (2020) afirmam que, a síndrome respiratória aguda grave (SARS-CoV-2) também conhecido como "novo coronavírus" foi declarada como pandemia, em março de 2020, sendo causada por um vírus que, segundo o Centro de Controle e Prevenção de Doenças (CDC), possui alta capacidade de transmissão entre as pessoas (CDC, 2020).

Sendo assim, o fechamento de fronteiras e de estabelecimentos, a intensificação dos cuidados com a saúde, quarentenas, restrições de viagens, triagem em aeroportos, e o isolamento social para impedimento de aglomerações de pessoas, vem sendo implementado por diversos continentes como medidas preventivas para conter a transmissão da SARSCoV-2 (Duddu, 2020; Salcedo; Cherelus, 2020; Zhou, 2020).

Ainda que, estas medidas sejam efetivas na redução da disseminação do novo coronavírus (Koo et al., 2020; Wells et al., 2020), os impactos globais causados pela COVID-19 são evidentes nas mais diversas áreas, principalmente socioeconômica (Scott, 2020).

Em decorrência ao estado de emergência durante a pandemia, a escassez de suprimentos e a consequente elevação dos preços, têm afetado diversos setores, principalmente no âmbito da saúde (Beraldo, 2020; Ulrich, 2020).

Segundo Azevedo e Menezes (2020), informes de escassez de suprimentos de produtos farmacêuticos têm se tornado cada vez mais frequentes no mundo. Assim, a necessidade de medidas assistenciais dos chefes de Estado de diversas nações vem sendo implementadas em diversos países, como por exemplo, o fechamento de empresas de setores não essenciais, resultando na crise comercial (Cowan, Morgan, \& Zengerle, 2020).

De acordo com a Organização Mundial de saúde (2020), a transmissão da COVID-19 pelo indivíduo infectado ocorre nos três primeiros dias após a manifestações dos sintomas, embora a disseminação possa ocorrer antes mesmo do surgimento destes e em estágios iniciais da doença.

A exposição ao vírus e o início dos sintomas (tempo de incubação) compreende um período de cinco dias, embora o intervalo consiga variar de 2 a 14 dias (CDC, 2020; Rothan; Byrareddy, 2020).

Entre as várias formas de transmissão da SARS-CoV-2, o Centro de Controle e Prevenção de Doenças considera pequenas gotículas expelidas durante as conversas, tosses, espirros, e o contato próximo entre indivíduos (CDC, 2020; OMS, 2020).

Falta de ar, tosse seca e febre, são os principais sintomas da COVID-19. Quadros severos da doença podem se desenvolver em alguns pacientes, sendo necessário a hospitalização. Pneumonia, insuficiência respiratória hipoxêmica e choque séptico compreendem as complicações mais frequentes da COVID-19 (CDC, 2020; Guan et al., 2020).

Visto que, o evento da COVID-19, ainda está sendo investigado, a difusão de conhecimentos sobre essa doença no contexto global se torna necessária. Assim, a importância deste estudo consiste em compreender as medidas preventivas para o combate à COVID-19, com uma abordagem para o impacto do isolamento social na sociedade. 
Almejamos que, este estudo possa contribuir para maior compreensão da população quanto as medidas preventivas, a fim de estabelecer estratégias para o combate a pandemia da COVID-19. Sendo assim, este estudo teve como objetivo identificar o impacto do isolamento social em tempos de COVID-19, e relatar as principais estratégias de combate à doença.

\section{Metodologia}

Trata-se, de um estudo de revisão bibliográfica baseado no método revisão integrativa da literatura, com abordagem qualitativa. Este método compreende uma ferramenta de investigação que permite à procura, a avaliação crítica e a síntese das evidências disponíveis sobre o tema investigado, em que o produto final é o estado do conhecimento, a implementação de intervenções efetivas na prestação de cuidados e na redução de custos. Além disso, permite a identificação de fragilidades, que poderão conduzir ao desenvolvimento de futuras investigações (Sousa, Marques-Vieira, Severino, \& Antunes, 2017).

A revisão consiste um tipo de revisão da literatura que reúne achados de estudos desenvolvidos mediante diferentes metodologias, permitindo aos revisores sintetizar resultados sem ferir a filiação epistemológica dos estudos empíricos incluídos. Portanto, para que esse processo concretize-se de maneira lógica, isenta de desatinos epistemológicos, a revisão requer que os revisores procedam à análise e à síntese dos dados primários de forma sistemática e rigorosa (Soares et al., 2014).

Este método de estudo requer um padrão de excelência quanto ao rigor metodológico para que seu produto possa trazer contribuições significativas para a ciência e para a prática clínica. A preservação deste padrão requer o uso de métodos que garantam a análise precisa, objetiva e completa do tema revisado; o suporte teórico para analisar resultados, métodos, sujeitos e variáveis dos estudos primários; a provisão de todas as informações contidas nos estudos revisados e não apenas os principais resultados, de modo a informar o leitor sem o sobrecarregar com informações desnecessárias (Soares et al., 2014).

Segundo Denzin e Lincoln (2006), a pesquisa qualitativa apresenta uma abordagem esclarecedora do mundo, ou seja, seus pesquisadores estudam as coisas em seus cenários naturais, tentando entender os fenômenos em termos dos significados que as pessoas a eles conferem.

O levantamento dos dados foram realizados entre os meses de março a novembro de 2020, por meio da busca de artigos indexados na Biblioteca Virtual em Saúde (BVS), e Scientific Electronic Library Online (SciELO), com o auxílio das seguintes bases de dados: PubMed (National Center for Biotechnology Information), Lilacs (Literatura Latino-Americana e do Caribe em Ciências da Saúde), Base de Dados de Enfermagem (BDENF).

No Portal da Biblioteca Virtual em Saúde (http://decs.bvs.br), foram localizados os seguintes Descritores em Ciências da Saúde: Infecções por Coronavirus, Prevenção de doenças; Isolamento Social; foi utilizado o operador booleano AND entre os descritores citados.

Os critérios de inclusão para a seleção dos conteúdos foram, artigos completos na linguagem portuguesa, inglesa e espanhola. Publicados na íntegra de acordo com a temática proposta, documentos, regulamentações, normativas de entidades de saúde, artigos, capítulos de livros publicados nos referidos bancos de dados compreendendo o ano de 2020.

Os critérios de exclusão, foram artigos duplicados, incompletos, resumos, resenhas, debates, relato de experiência, artigos publicados em anais de eventos e indisponíveis na íntegra. 


\section{Resultados e Discussão}

A partir da revisão de literatura e análise dos estudos indexados nas bases de dados eletrônicas, acerca da temática proposta, foram encontrados 400 estudos científicos, sendo que, apenas 52 estudos foram selecionados, 27 atenderam aos critérios de inclusão previamente estabelecidos, destes, 22 foram excluídos com base nos critérios de exclusão. Restando 05 artigos para composição e análise do estudo.

Além destes, foram analisados 04 estudos referentes à entidades de saúde indexados nas bases de dados eletrônicas, acerca da temática proposta, sendo (Organização Mundial de Saúde; Organização Pan-Americana de Saúde; Ministério da Saúde; Secretaria de Saúde). O detalhamento das etapas de pesquisa está apresentado a seguir na Figura 1.

Figura 1. Fluxograma de identificação e seleção dos artigos. 2020.

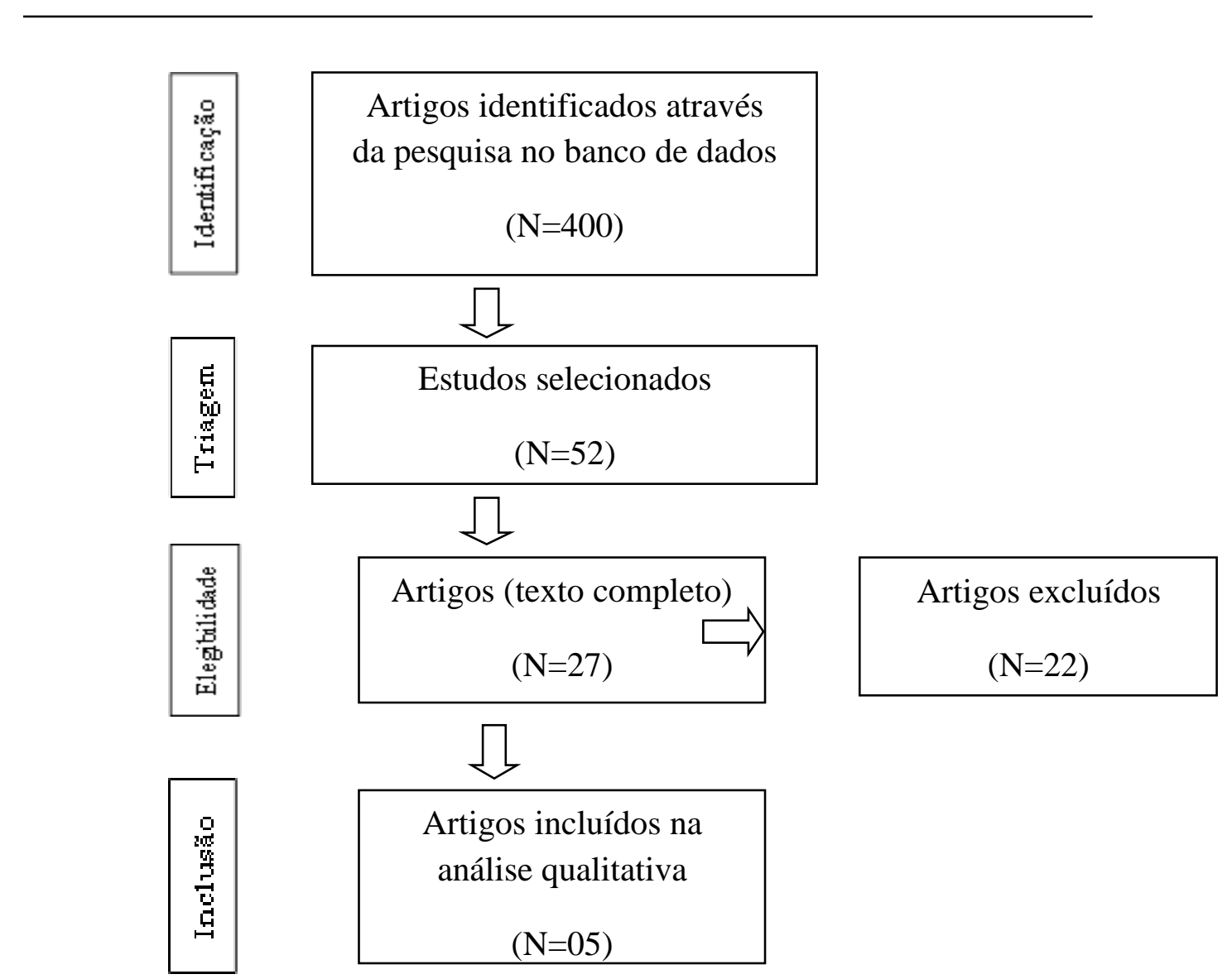

Fonte: Autores (2020).

A partir da revisão de literatura e análise dos estudos indexados nas bases de dados eletrônicas acerca da temática proposta, podemos destacar que, são várias as estratégias e recomendações que visam prevenir a transmissão da COVID-19.

O termo "vírus" vem do latim virus, compreendido como "veneno" ou "toxina". Dispõe sua maioria de 20-300 nm de diâmetro, portam um genoma composto de uma ou diversas moléculas de ácido nucléico (DNA ou RNA), revestidos por um envoltório proteico formado por várias proteínas, e por um complexo envelope em uma bicamada lipídica (Chang, Yan, \& Wang, 2020). 
O coronavírus consiste em um vírus de RNA com perspectiva positiva, são envelopados, e portam uma estratégia de replicação rara, fato que, possibilita a variação de patogenicidade e facilidade de amoldamento em discrepantes ambientes (Chang, Yan \& Wang, 2020).

O SarsCoV-2 deriva-se de uma nova espécie identificada em dezembro de 2019, e por não ter sido isolada, as medidas a serem implementadas para o enfrentamento da pandemia almejam em si o aniquilamento do vírus, impedindo sua rápida transmissão de pessoa a pessoa (OMS, 2020 \& Shang, et al, 2020).

Visto a aparição dos primeiros diagnósticos de COVID-19 no país, os casos eram majoritariamente importados e a estratégia de contenção da epidemia baseava-se na busca e isolamento dos casos e contatos, para se esquivar-se transmissão do vírus de pessoa a pessoa, de conduta sustentada. Com o avanço do número de casos da COVID-19 e a ocorrência de transmissão comunitária, estratégias de mitigação traspassaram a ser adotadas, buscando-se evitar a ocorrência de casos críticos e óbitos pela doença. Tais estratégias engloba medidas de precaução hospitalar para os casos graves, além de medidas de isolamento para casos brandos e contatos (Brasil, 2020).

A transmissão do Sars-CoV-2 de pessoa a pessoa se profere por meio da autoinoculação do vírus em membranas mucosas (nariz, olhos ou boca) e do contato com superfícies contaminadas, o que vem chamando cada vez mais atenção para a necessidade de adoção rápida e preventiva de parâmetros de proteção humana a fim de impossibilitar o contágio de pessoas (Kampf, G. et al., 2020).

Deste modo, uma das medidas mais importantes para a prevenção da transmissão refere - se, higiene das mãos, julgada uma medida de baixo custo e alta efetividade contra o vírus, por serem as mãos o principal veículo de contaminação corporal. Embora inúmeros trabalhos apontem para a indevida adoção dessa prática entre os profissionais durante o auxílio de pacientes nos serviços de saúde (Kampf, et al., 2020).

A prática da higienização das mãos pela ação de fricção com água e sabão diminui um pouco a ocorrência das infecções preveníveis, reduzindo a morbimortalidade em serviços de saúde (Amorim, et al., 2018 \& OMS, 2020).

O Centro de Controle e Prevenção de Doenças destaca a lavagem das mãos e o isolamento social como as principais medidas de prevenção à COVID-19. A lavagem das mãos deve ocorrer com água e sabão, ou desinfetante à base de etanol a pelo menos $60 \%$ ou isopropanol a $70 \%$. Não sendo recomendado tocar nos olhos, nariz ou boca antes da higienização das mãos. Já o isolamento social deve ocorrer principalmente para indivíduos que viajaram recentemente para um país ou região com transmissão generalizada, bem como, para aqueles que estão com suspeita de infecção, ou que testaram positivo para a COVID-19 (CDC, 2020).

Ainda considerando as estratégias de combate à COVID-19, Kampf et al. (2020) apresentam em seus estudos que, a limpeza de superfícies deve ocorrer com etanol (62 a 71\%) e hipoclorito $(0,1 \%)$.

Por sua vez, a Organização Mundial de Saúde (2020), recomendou algumas medidas preventivas a COVID-19, sendo, o uso de máscaras faciais, a cobertura do nariz e boca com o cotovelo dobrado, ou o uso de lenço de papel ao tossir ou espirrar, além do distanciamento físico entre os indivíduos através de estratégias que incluem quarentenas, restrições de viagem, fechamento de escolas, universidades, e estabelecimentos de aglomeração (OMS, 2020).

Em outras palavras, a Organização Mundial de Saúde (2020) afirma que, as estratégias de controle da transmissão da COVID-19 consiste na contenção, mitigação e na supressão. A contenção compreende a fase inicial do surto, ou seja, a implementação de medidas estratégicas que visam rastrear, e isolar os indivíduos infectados (OMS, 2020).

A mitigação é a fase em que não é possível conter a propagação da doença. Essa fase tem como objetivo reduzir o número de contaminações pela COVID-19, e diminuir o risco de sobrecarga do sistema de saúde. Assim, são implementadas medidas preventivas que visam a diminuição da circulação de pessoas, como, o cancelamento de eventos e a paralisação das aulas (Zhang \& Qian, 2020). 
Já a supressão tem como objetivo reduzir o número de casos suspeitos e confirmados da COVID-19, por meio de medidas como, o isolamento social e a fiscalização de órgãos públicos (Ferguson et al., 2020).

Ainda nos estudos de Ferguson et al. (2020), alguns especialistas defendem que, a supressão compreende a medida estratégica mais eficiente no controle da pandemia da COVID-19, uma vez que, a demanda da população nos Serviços de Saúde durante o pico das infecções pode reduzir em aproximadamente dois terços.

Até o momento, não existem medicamentos ou outras medidas terapêuticas para combater a COVID-19. Contudo, várias pesquisas têm sido desenvolvidas nesse sentido, assim como a avaliação e investigação da eficácia de medicamentos já existentes, para atuação no combate ao novo coronavírus (Mccreary, Pogue \& Pharmacists, 2020).

Em relação aos medicamentos já existentes, a testagem com a cloroquina e a hidroxicloroquina foram suspensas após a realização de um estudo internacional com 96.032 pessoas hospitalizadas com a COVID-19. O estudo constatou que o uso de ambas as drogas não apresentava benefícios aos pacientes, e aumentava o risco de arritmias cardíacas e morte (Mehra et al., 2020).

\section{Considerações Finais}

O estudo evidenciou a importância das recomendações de medidas preventivas para a minimização do impacto do isolamento social na sociedade em tempos de COVID-19. Segundo os estudos de Craveiro (2020), os países que adotaram medidas de distanciamento social precocemente, fizeram rastreamento de usuários infectados, testagem em massa para a identificação do vírus, e ofereceram suporte hospitalar adequado, tiveram melhores resultados na luta contra a COVID-19, a Taiwan, Coreia do Sul, Islândia e Alemanha são exemplos de países que adotaram essas medidas precocemente e alcançaram o achatamento da curva pandêmica, apresentando redução do número de mortes e casos confirmados pela doença.

Em contrapartida, além da flexibilização das medidas preventivas em outros países, também vem ocorrendo a falta de definição de estratégias unificadas e falta de consenso entre as autoridades, este cenário de incertezas, vem dificultando o enfrentamento da COVID-19 no pais, assim, ainda é difícil prever quando a disseminação da doença será completamente controlada (Moraes, 2020).

\section{Referências}

Amorim, C. S. V., Pinheiro, I. F., Vieira, V. G., Guimarães, R. A., Nunes, O. S., \& Marinho, T. A. (2020). Hand hygiene and influenza prevention: knowledge of health students; 27(4):e4570017.

Azevedo, A. L., \& Menezes, M. (2020). Coronavírus: falta de insumos e medo de contágio deixam médicos e enfermeiros apreensivos.

Beraldo, L. (2020). Covid-19: Cade investiga aumento abusivo de preços de produtos.

Centro de Controle e Prevenção de Doenças (CDC). (2020). Coronavirus Disease 2019 (COVID-19).

Centro de Controle e Prevenção de Doenças (CDC). (2020). Coronavirus Disease 2019 (COVID-19): Information for Laboratories.

Centro de Controle e Prevenção de Doenças (CDC). (2020). Coronavirus Disease 2019 (COVID-19): Hand Hygiene Recommendations.

Centro de Controle e Prevenção de Doenças (CDC). (2020). Coronavirus Disease 2019 (COVID-19): how easily the virus spreads. USA,

Centro de Controle e Prevenção de Doenças (CDC). (2020). Coronavirus Disease 2019 (COVID-19): How to Protect Yourself \& Others.

Centro de Controle e Prevenção de Doenças (CDC). (2020). Coronavirus Disease 2019 (COVID-19): Interim Clinical Guidance for Management of Patients with Confirmed Coronavirus Disease (COVID-19). EUA.

Centro de Controle e Prevenção de Doenças (CDC). (2020). Preliminary Estimates of the Prevalence of Selected Underlying Health Conditions Among Patients with Coronavirus Disease 2019-United States.

Chang, L., Yan, Y., \& Wang, L. (2020). Coronavirus disease 2019: Coronaviruses and blood safety. Transfus Med Rev [Internet].

Cowan, R., Morgan, D., \& Zengerle, P. (2020). Covid-19: Senado dos EUA aprova plano de US\$2tri de combate à crise. 
Research, Society and Development, v. 10, n. 10, e57910111281, 2021

(CC BY 4.0) | ISSN 2525-3409 | DOI: http://dx.doi.org/10.33448/rsd-v10i1.11281

Duddu, P. (2020). Coronavirus outbreak: safety measures at major international airports.

Ferguson, N. M. et al. (2020). Impact of non-pharmaceutical interventions (NPIs) to reduce COVID19 mortality and healthcare demand.

Gorbalenya, A. E. et al. (2020). The species Severe acute respiratory syndrome-related coronavirus: classifying 2019-nCoV and naming it SARS-CoV-2. Nature Microbiology, 5(4), 536-544.

Guan, W. J. et al. (2020). Clinical Characteristics of Coronavirus Disease 2019 in China. New England Journal of Medicine.

Jhu. (2020). COVID-19 Dashboard by the Center for Systems Science and Engineering (CSSE) at Johns Hopkins University (JHU).

Kampf, G. et al. (2020). Persistence of coronaviruses on inanimate surfaces and their inactivation with biocidal agents. Journal of Hospital Infection, 104(3), 246251.

Kampf, G., Todt, T., Pfaender, S. \& Steinmann, E. (2020). Persistence of coronaviruses on inanimate surfaces and their inactivation with biocidal agents. J Hosp Infect [Internet].

Koo, J. R. et al. (2020). Interventions to mitigate early spread of SARS-CoV-2 in Singapore: a modelling study. The Lancet Infectious Diseases.

Mccreary, E. K., Pogue, J. M., Pharmacists, O. B. O. T. S. O. I. D. (2020). Coronavirus Disease 2019 Treatment: A Review of Early and Emerging Options. Open Forum Infectious Diseases, 7(4), 2020.

Mehra, M. R., et al. (2020). Hydroxychloroquine or chloroquine with or without a macrolide for treatment of COVID-19: a multinational registry analysis. The Lancet.

Ministério da Saúde. Boletim epidemiológico-SVS. Infecção Humana pelo novo Coronavírus (N COV-2019). Ministério da saúde, Brasília.

Ministério da Saúde. Protocolo de manejo clínico para o coronavírus (2019-nCoV), Ministério da saúde, Brasília.

Rothan, H. A., \& Byrareddy, S. N. (2020). The epidemiology and pathogenesis of coronavirus disease (COVID-19) outbreak. Journal of Autoimmunity, p. $102433,2020$.

Salcedo, A., \& Cherelus, G. (2020). Coronavirus Travel Restrictions, Across theGlobe.

Scott, J. (2020). The economic, geopolitical and health consequences of COVID-19.

Shang, J., Wan, Y., Liu, C., Yount, B., Gully, K., Yang, Y., et al. (2020). Structure of mouse coronavirus spike protein complexed with receptor reveals mechanism for viral entry. PLoS Pathog [Internet].

Ulrich, K. (2020). Efeitos do coronavírus sobre as cadeias mundiais de abastecimento.

Wells, C. R. et al. (2020). Impact of international travel and border control measures on the global spread of the novel 2019 coronavirus outbreak. Proceedings of the National Academy of Sciences, 117(13), 7504-7509.

Word Health Organization [OMS]. (2020). Considerations for quarantine of individuals in the context of containment for coronavirus disease (COVID-19): Interim guidance [Internet]. Geneva $(\mathrm{CH})$.

World Health Organization [OMS]. (2020). Critical preparedness, readiness and response actions for COVID-19 [Internet]. Geneva (CH).

Zhang, W., \& Qian, B.-y. (2020). Making decisions to mitigate COVID-19 with limited knowledge. The Lancet Infectious Diseases.

Zhou, F. et al. (2020). Clinical course and risk factors for mortality of adult inpatients with COVID- 19 in Wuhan, China: a retrospective cohort study. The Lancet, 395(10229), 1054-1062, 2020.

Zhou, P. et al. (2020). Discovery of a novel coronavirus associated with the recent pneumonia outbreak in humans and its potential bat origin. bioRxiv, p. 2020.2001.2022.914952, 2020.

Zhou, V. (2020). Coronavirus: Shanghai neighbour Zhejiang imposes draconian quarantine.

Zou, L. et al. (2020). SARS-CoV-2 Viral Load in Upper Respiratory Specimens of Infected Patients. New England Journal of Medicine, 382(12), 1177-1179, 2020. 